\title{
10 Gb/s Radio-Over-Fiber at 28 GHz Carrier Frequency Link Based on 1550 nm VCSEL Chirp Enhanced Intensity Modulation after 2 km Fiber
}

\author{
Joris Van Kerrebrouck ${ }^{1}$, Haolin Li ${ }^{1}$, S. Spiga ${ }^{2}$, Markus C. Amann ${ }^{2}$, Xin Yin ${ }^{1}$, Johan \\ Bauwelinck $^{1}$, Piet Demeester ${ }^{1}$, Guy Torfs ${ }^{1}$ \\ ${ }^{1}$ IDLab, INTEC, Ghent University - imec, Ghent, Belgium. \\ ${ }^{2}$ Walter Schottky Institut, Munich, Germany.
}

\begin{abstract}
Gb} / \mathrm{s}, 28 \mathrm{GHz}$ radio-over-fiber transmission using a directly-modulated single-mode C-band VCSEL is demonstrated over $2 \mathrm{~km}$. The chirp of the VCSEL is translated into intensity modulation to extend the fiber-reaches and increase the power budget with $10 \mathrm{~dB}$.
\end{abstract}

OCIS codes: $060.2330,140.7270$.

\section{Introduction}

To support the foreseen high data throughput in next-generation mobile networks (5G), both spatial densification and higher frequencies bands are envisioned. Especially $\mathrm{mmWave}$ frequencies are gaining traction thanks to the available bandwidth at these frequencies. Recent trials propose $28 \mathrm{GHz}$ as the mmWave band for mobile communication [1,2]. However, to overcome the higher path loss at these frequencies, higher cellular density is required with multiple small radio cells, which demands a very cheap and simple design for the remote antenna unit (RAU) [3]. Directly modulated Vertical Cavity Surface Emitting Lasers (VCSELS) are cost-friendly, simple and easy to fabricate, an ideal candidate for Radio-Over-Fiber (ROF) networks. Compared to distributed feedback laser (DFB), which typically consumes $100 \mathrm{~mA}$, the relatively high bandwidth and the low driving currents $(10 \mathrm{~mA})$ are definitely benefits.

The modulation bandwidth of an intensity modulation / direct detection (IM-DD) optical link, is heavily dependent on the fiber length due to chromatic dispersion of standard single-mode fiber (SSMF). To make it even worse, the chirp introduced by directly modulating the laser reduces the bandwidth of the link even further. Optical single-side band (SSB) modulation with a single carrier modulation is insensitive to chromatic dispersion. However, SSB transmitters require a complex external IQ modulator or sharp optical filters. To reduce the chirp, optical injection locking (OIL) has been demonstrated. OIL can greatly enhance the modulation bandwidth of a directly-modulated laser. However, it comes with the extra cost of an additional laser (master laser), which is typically a better and more expensive DFB laser with a very narrow linewidth. Furthermore, an optical circulator is needed, further increasing the cost and complexity of the link.

Due to chromatic dispersion in standard single-mode fiber, each optical frequency travels at a different velocity. As a result, intensity modulated signals are transformed into phase modulation, but also the opposite is true: Chromatic dispersion translates the parasitic phase modulation (PM, chirp) into an intensity modulation (IM). Our experiment confirms this prediction and shows that chirp in combination with the chromatic dispersion can even increase the transmission gain at higher frequency bands, surpassing the gain of a back-to-back (B2B) transmission at DC. VCSELs are far more efficient in phase modulation than in intensity modulation [4]. For example, this is intentionally used in [5], where they utilize a Mach-Zehnder interferometer (MZI) to convert the large phase modulation to intensity modulation. Chromatic dispersion can realize a similar effect, resulting in a signal gain that can be used to unlock higher frequency bands or to overcome the splitting ratio in passive optical networks (PON). The required amount of chromatic dispersion to realize this effect is frequency dependent, as a result, this technique is only attractive for narrow band modulations such as ROF, but less suited for typical broadband applications.

\section{VCSEL and chirp measurements}

The used VCSEL [6] is a $1550 \mathrm{~nm}$ long wavelength single-mode VCSEL. Back-to-back S21 transmission measurements are depicted in Fig. 1a for different bias currents. The InP-based VCSEL uses an ultra-short semiconductor cavity and two distributed Bragg reflectors (DBRs) to enhance its direct modulation bandwidth to $23 \mathrm{GHz}$. The static 


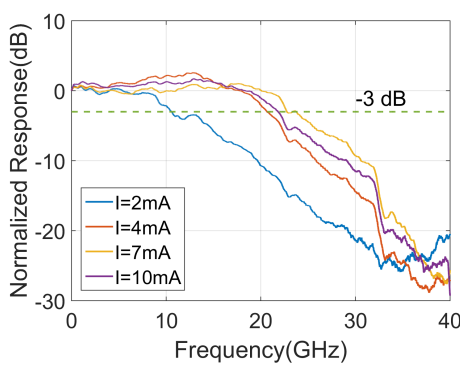

(a)

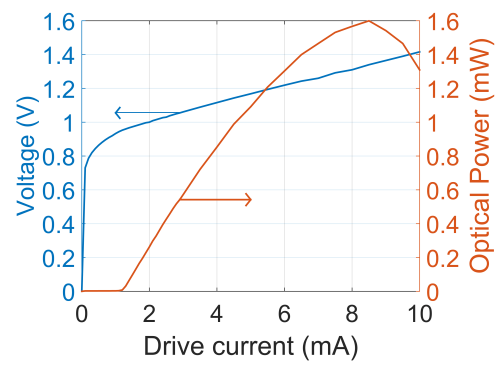

(b)

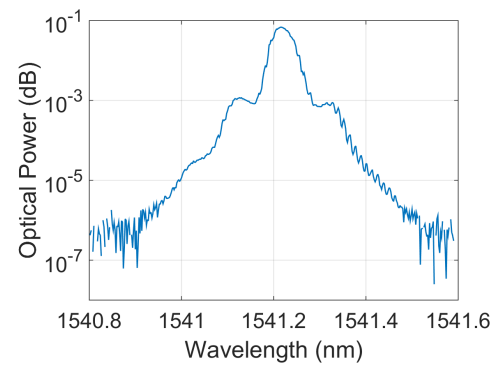

(c)

Fig. 1: Characteristics of the single mode 1550nm VCSEL: (a) S21 measurements, (b) power-current-voltage (PIV) graph and (c) optical spectrum of the VCSEL.

P-I-V curve of the VCSEL is shown in Fig. $1 \mathrm{~b}$ and the optical spectrum is shown in Fig. 1c. The spectrum is measured while operating the VCSEL with a bias current of $7 \mathrm{~mA}$ without any temperature control. In this case, the static chirp is $0.56 \mathrm{~nm} / \mathrm{mW}$ or $70 \mathrm{GHz} / \mathrm{mW}$. The following measurements are also performed using this bias current.

\section{Chirp enhanced intensity modulation}

The transmission parameters of a complete optical link with different fiber lengths are shown in Fig. 2a. This optical link includes the electrical probe, VCSEL, fiber and a $40 \mathrm{GHz}$ photodiode. It is evident that fiber lengths of 2 and $3 \mathrm{~km}$ result in an additional gain of more than $10 \mathrm{~dB}$ at frequencies above $30 \mathrm{GHz}$ compared to the back-to-back (B2B) measurement. For $3 \mathrm{~km}$ fiber, the optical link has even more transmission gain at $20 \mathrm{GHz}$ than at DC. To calibrate out all the parasitic effects of the probe and photodiode, the S21 parameters of various fiber lengths are normalized to that in B2B transmission. The normalization is shown in Fig.2b and isolates the effect of the interplay of intensity modulation, phase modulation and chromatic dispersion. The measurements are only valid until $50 \mathrm{GHz}$, where the measured S21 parameters (Fig. 2a) disappear in the noise floor.

This effect is easily predictable: in Matlab, we created an IM and PM signal for modulation frequencies from 1 to $60 \mathrm{GHz}$ as shown in Eq. 1, where $\omega_{m}$ and $\omega_{c}$ represent the modulation and optical carrier angular frequency, respectively. $A$ is the depth of the IM and $\beta$ is the gain of the PM. For small-signal modulation above $2 \mathrm{GHz}$, the IM depth $A$ and PM gain $\beta$ are linearly proportional to each other. Specifically, for this VCSEL, the factor $\frac{2 \beta}{A}$ directly equals the laser's $\alpha_{H}$-factor $\left(\alpha_{H}=\frac{2 \beta}{A}=12\right)$ [7]. The simulation is fine-tuned by fitting the phase $(\phi=0.877 \mathrm{rad})$ and delay $(\tau=4.7 \mathrm{ps})$ between the phase modulation and intensity modulation. This signal is then deformed by a chromatic dispersion filter to mimic the fiber length with a chromatic dispersion coefficient of $16 \mathrm{ps} /(\mathrm{nm} \mathrm{km})$ and an attenuation of $0.2 \mathrm{~dB} / \mathrm{km}$. An optical connector loss of $0.5 \mathrm{~dB} /$ connector is also included in the simulation. Next, this signal is detected by capturing its envelope. To obtain the chirp enhanced IM factor, the power of the detected signal is normalized to the power detected after B2B transmission. The results of this simulation are shown as the dashed lines in Fig. $2 b$ and closely follow the measurements.

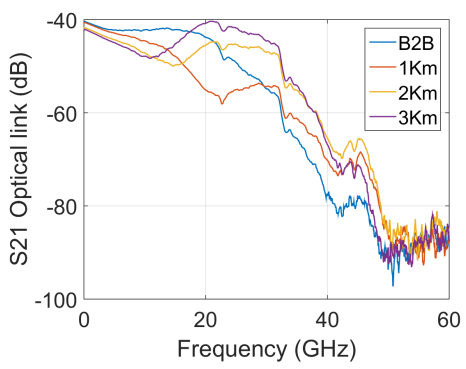

(a)

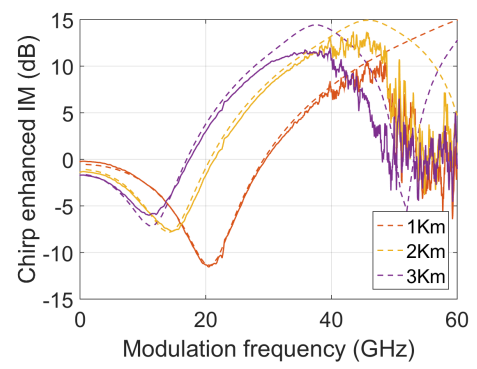

(b)

Fig. 2: (a) S21 of the total optical link including electrical probe, VCSEL, fiber, and photodiode. (b) The normalized (to B2B transmission) enhancement of the intensity modulation signal due to chirp and chromatic dispersion.

$$
s(t)=\underbrace{\left(1+A \cos \left(\omega_{m}(t-\tau)\right)\right)}_{\text {Intensity modulation }} \cos (\omega_{c} t+\underbrace{\beta \cos \left(\omega_{m} t-\phi\right)}_{\text {Phase modulation }})
$$




\section{Experimental setup and results}

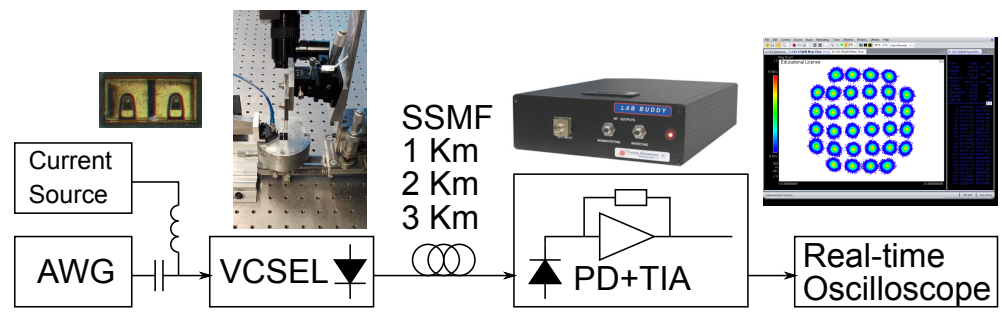

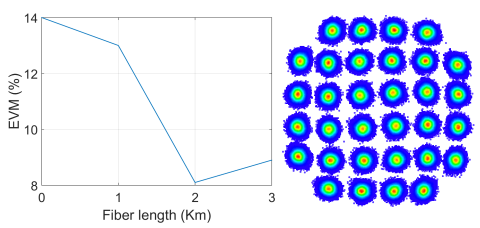

(b) (c)

(a)

Fig. 3: (a) Experimental setup. (b) EVM measurements of a 32-QAM 2 GBaud modulated at $28 \mathrm{GHz}$ after directly modulated ROF transmission. (c) Received constellation diagram of a 32-QAM 2 GBaud at $28 \mathrm{GHz}$ after $2 \mathrm{~km} \mathrm{SSMF}$.

To verify this effect in the context of mmWave ROF transmission, a 32QAM 2 GBaud signal modulated at $28 \mathrm{GHz}$ was transmitted over different SSMFs with fiber lengths of 1,2 and $3 \mathrm{~km}$, resulting in a total data rate of $10 \mathrm{~Gb} / \mathrm{s}$. The experimental setup is depicted in Fig. 3a. The signal was generated with an arbitrary waveform generator (AWG)(Keysight M8196A $92 \mathrm{GSa} / \mathrm{s}$ ) and was, together with a DC current of $7 \mathrm{~mA}$, applied to the VCSEL with an electrical probe. A lensed fiber was used to capture the optical signal. After transmission through the SSMF, a photodiode with a TIA (Discovery lab buddy DSC-R409) with $30 \mathrm{GHz}$ bandwidth was used as an O/E converter. The detected signal was captured with a real-time oscilloscope (DSAZ634A) and Keysight VSA software decoded the single carrier data and measured the error vector magnitude (EVM). Fig. 3b shows the RMS EVM for the complete optical link using B2B $(0 \mathrm{~km}), 1,2$ and $3 \mathrm{~km}$ fiber length. It is notable that the EVM decreases with a longer fiber length as predicted by the theory described above. $2 \mathrm{~km}$ fiber performs the best with an EVM of 8.02\% (i.e. -21.94 $\mathrm{dB})$. The constellation diagram of the received symbols after $2 \mathrm{~km}$ transmission is shown in Fig. $3 \mathrm{c}$.

\section{Conclusion}

The paper presents the effectiveness of phase modulation originated from the parasitic chirp in the VCSELs. The use of $1550 \mathrm{~nm}$ wavelength VCSEL opens the possibility to counteract chromatic dispersion in fiber by converting the parasitic phase modulation to intensity modulation. We have demonstrated that the chirp enhanced intensity modulation boosts the transmission gain with $10 \mathrm{~dB}$ at $28 \mathrm{GHz}$ compared with the IM-based optic link, allowing $10 \mathrm{~Gb} / \mathrm{s} \mathrm{ROF}$ transmission.

\section{Acknowledgements}

This authors would like to acknowledge the support of the ERC advanced grant 695495 ATTO: A new concept for ultra-high capacity wireless networks.

\section{References}

1. Kei Sakaguchi et al., "Where, When, and How mmWave is Used in 5G and Beyon," Computing Research Repository, arXiv:1704.08131.

2. M. Ptzold, "Fifth-Generation Technology Offers Trillion-Dollar Business Opportunities [Mobile Radio]," in IEEE Vehicular Technology Magazine, vol. 12, no. 3, pp. 4-11, Sept. 2017.

3. B. Lannoo et al., "Radio-over-fibre for ultra-small 5G cells," 201517 th International Conference on Transparent Optical Networks (ICTON), Budapest, 2015, pp. 1-4.

4. J. A. Altabas, D. Izquierdo, J. A. Lazaro and I. Garces, "1.25-2.5Gbps cost-effective transceiver based on directly phase modulated VCSEL for flexible access networks," 2017 Optical Fiber Communications Conference and Exhibition (OFC), Los Angeles, CA, 2017, pp. 1-3.

5. Z. Yang et al., "High-gain phase modulated analog photonic link using high-power balanced photodiodes," 2017 Optical Fiber Communications Conference and Exhibition (OFC), Los Angeles, CA, 2017, pp. 1-3.

6. S. Spiga et al., "Single-Mode High-Speed 1.5-um VCSELs," in Journal of Lightwave Technology, vol. 35, no. 4, pp. 727-733, Feb.15, 152017.

7. J. G. Provost and F. Grillot, "Measuring the Chirp and the Linewidth Enhancement Factor of Optoelectronic Devices with a MachZehnder Interferometer," in IEEE Photonics Journal, vol. 3, no. 3, pp. 476-488, June 2011. 